JOURNAL OF

SYMPLECTIC GEOMETRY

Volume 11, Number 2, 163-166, 2013

\title{
SYMPLECTIC RIGIDITY AND WEAK COMMUTATIVITY
}

\author{
Franco Cardin and Simone Vazzoler
}

\begin{abstract}
We present a new and simple proof of Eliashberg-Gromov's theorem based on the notion of $C^{0}$-commutativity introduced by Cardin and Viterbo in $[\mathbf{1}]$.
\end{abstract}

\section{Introduction}

The notion of $C^{0}$-commutativity has been introduced in $[\mathbf{1}]$ in connection with the problem of finding variational solutions of multi-time HamiltonJacobi equations. The proof of the main theorem is essentially based on the use of Viterbo's capacities. In that paper, it has been foreseen that from the $C^{0}$-commutativity framework Eliashberg-Gromov's theorem on symplectic rigidity could follow. An interesting proof of this fact has been recently worked out by Humilière (see [4]) using the concept of pseudorepresentations.

The question of the $C^{0}$-closure of the group of symplectomorphisms is widely considered as the starting point of the study of symplectic topology; for this reason is important to enrich this particular area with new proofs, eventually trying to simplify the subject. This note presents a new proof of Eliashberg-Gromov's theorem, starting from the concept of $C^{0}$ commutativity and using simple algebraic arguments.

\section{Weak commutativity and Eliashberg-Gromov's theorem}

In the following, by a function of class $C^{1,1}$ we mean a $C^{1}$ function with Lipschitz derivative.

Definition 1. Let $H, K$ be two autonomous Hamiltonians. We will say that $H$ and $K C^{0}$-commute if there exist two sequences $H_{n}, K_{n}$ of $C^{1}$ Hamiltonians $C^{0}$-converging to $H$ and $K$, respectively, such that, in the $C^{0}$-topology:

$$
\lim _{n \rightarrow \infty}\left\{H_{n}, K_{n}\right\}=0 .
$$


The previous definition is a good extension of the standard Poisson brackets since the following theorem holds.

Theorem 2 (Cardin, Viterbo [1]). Let $H$ and $K$ be two compactly supported Hamiltonians of class $C^{1,1}$. If they $C^{0}$-commute then $\{H, K\}=0$ in the usual sense.

The following lemma is the generalization of Theorem 2 to the affine at infinity case.

Lemma 3 (Humilière, [4]). Let $u, v$ two affine maps $\mathbb{R}^{2 n} \rightarrow \mathbb{R}$ and $H_{n}, K_{n}$ be compactly supported Hamiltonians, such that

$$
H_{n} \rightarrow H, K_{n} \rightarrow K,\left\{H_{n}+u, K_{n}+v\right\} \rightarrow 0 .
$$

Then $\{H+u, K+v\}=0$.

In what follows, we will consider only sequences of compactly supported symplectomorphisms $\Phi^{(n)}$, more precisely such that $\operatorname{supp}\left(\Phi^{(n)}-\right.$ Id) is compact.

Theorem 4 (Symplectic rigidity, $[2,3]$ ). The group of compactly supported symplectomorphisms is $C^{0}$-closed in the group of all diffeomorphisms of $\mathbb{R}^{2 d}$.

Proof. To fix the notations: $(q, p)=\left(q_{1}, \ldots, q_{d}, p_{1}, \ldots, p_{d}\right) \in \mathbb{R}^{2 d}$ and denote by

$$
\left(Q_{1}^{(n)}(q, p), \ldots, Q_{d}^{(n)}(q, p), P_{1}^{(n)}(q, p), \ldots, P_{d}^{(n)}(q, p)\right),
$$

a sequence of symplectic transformations $C^{0}$-converging to

$$
\left(Q_{1}(q, p), \ldots, Q_{d}(q, p), P_{1}(q, p), \ldots, P_{d}(q, p)\right) .
$$

Note that we have to prove only $\left\{Q_{i}, P_{i}\right\}=1$. In fact, the other relations $\left\{Q_{i}, Q_{j}\right\}=0=\left\{P_{i}, P_{j}\right\}$, and $\left\{Q_{i}, P_{j}\right\}=0$ for $i \neq j$, are automatically satisfied in view of Lemma 3. Now we define a new sequence (using the previous one)

$$
\left\{\begin{array}{l}
\widetilde{Q}_{i}^{(n)}=Q_{i}^{(n)}+\frac{1}{\sqrt{d}} \sum_{k=1}^{d} P_{k}^{(n)}, \\
\widetilde{P}_{i}^{(n)}=P_{i}^{(n)}+\frac{1}{\sqrt{d}} \sum_{k=1}^{d} Q_{k}^{(n)}
\end{array}\right.
$$


that will $C^{0}$-converge to

$$
\left\{\begin{array}{l}
\widetilde{Q}_{i}=Q_{i}+\frac{1}{\sqrt{d}} \sum_{k=1}^{d} P_{k} \\
\widetilde{P}_{i}=P_{i}+\frac{1}{\sqrt{d}} \sum_{k=1}^{d} Q_{k}
\end{array}\right.
$$

Clearly $\left\{\widetilde{Q}_{i}^{(n)}, \widetilde{P}_{i}^{(n)}\right\}=0$, in fact

$$
\begin{aligned}
\left\{\widetilde{Q}_{i}^{(n)}, \widetilde{P}_{i}^{(n)}\right\}= & \left\{Q_{i}^{(n)}, P_{i}^{(n)}\right\}+\frac{1}{\sqrt{d}} \sum_{k=1}^{d}\left(\left\{P_{k}^{(n)}, P_{i}^{(n)}\right\}+\left\{Q_{i}^{(n)}, Q_{k}^{(n)}\right\}\right) \\
& +\frac{1}{d} \sum_{k=1}^{d}\left\{P_{k}^{(n)}, Q_{k}^{(n)}\right\}=1-1=0 .
\end{aligned}
$$

Again using Lemma 3 , we obtain $\left\{\widetilde{Q}_{i}, \widetilde{P}_{i}\right\}=0$ : passing to the limit,

$$
\begin{aligned}
\left\{\widetilde{Q}_{i}, \widetilde{P}_{i}\right\} & =\left\{Q_{i}, P_{i}\right\}+\frac{1}{\sqrt{d}} \sum_{k=1}^{d}\left(\left\{P_{k}, P_{i}\right\}+\left\{Q_{i}, Q_{k}\right\}\right)+\frac{1}{d} \sum_{k=1}^{d}\left\{P_{k}, Q_{k}\right\} \\
& =\left\{Q_{i}, P_{i}\right\}+\frac{1}{d} \sum_{k=1}^{d}\left\{P_{k}, Q_{k}\right\}=0 .
\end{aligned}
$$

Define (just to simplify the notations) $C_{i}(q, p)=\left\{Q_{i}, P_{i}\right\}$. For every fixed $(q, p) \in \mathbb{R}^{2 d}$, the last homogeneous linear system reads

$$
\left(\begin{array}{cccc}
d-1 & -1 & \ldots & -1 \\
-1 & d-1 & \ldots & -1 \\
\vdots & \vdots & \ddots & \vdots \\
-1 & -1 & \ldots & d-1
\end{array}\right)\left(\begin{array}{c}
C_{1} \\
C_{2} \\
\vdots \\
C_{d}
\end{array}\right)=\left(\begin{array}{c}
0 \\
0 \\
\vdots \\
0
\end{array}\right)
$$

This system has $C_{1}=C_{2}=\cdots=C_{d}$ as solution; in fact, the $d \times d$ matrix has determinant equal to zero: if we sum the last $d-1$ rows we get the opposite of the first row; in particular the rank of the matrix is $d-1$, so the subspace of solutions has dimension 1 and is exactly the space of equal components vectors. Recalling the Jacobi identity

$$
\{f,\{g, h\}\}+\{g,\{h, f\}\}+\{h,\{f, g\}\}=0,
$$

we obtain, considering terms like $\left\{Q_{i},\left\{Q_{j}, P_{j}\right\}\right\}$ for $i \neq j$,

$$
0=\left\{Q_{i},\left\{Q_{j}, P_{j}\right\}\right\}+\left\{Q_{j},\left\{P_{j}, Q_{i}\right\}\right\}+\left\{P_{j},\left\{Q_{i}, Q_{j}\right\}\right\}
$$

and since $\left\{Q_{i}, P_{j}\right\}=\left\{Q_{i}, Q_{j}\right\}=0$, we get

$$
\left\{Q_{i},\left\{Q_{j}, P_{j}\right\}\right\}=0 .
$$


In the same way, considering $\left\{P_{i},\left\{Q_{j}, P_{j}\right\}\right\}$ we obtain

$$
\left\{P_{i},\left\{Q_{j}, P_{j}\right\}\right\}=0 \text {. }
$$

Once we have set $C_{1}(q, p)=C_{2}(q, p)=\cdots=C_{d}(q, p)=C(q, p)$, using the previous relations, we have

$$
\left\{\begin{array}{c}
\left\{Q_{1}, C\right\}=0 \\
\left\{Q_{2}, C\right\}=0 \\
\quad \vdots \\
\left\{P_{d-1}, C\right\}=0 \\
\left\{P_{d}, C\right\}=0,
\end{array}\right.
$$

which is a homogeneous linear system of the type $A \cdot D C=0$

$$
\left(\begin{array}{cccccc}
-Q_{1, p_{1}} & \ldots & -Q_{1, p_{d}} & Q_{1, q_{1}} & \ldots & Q_{1, q_{d}} \\
-Q_{2, p_{1}} & \ldots & -Q_{2, p_{d}} & Q_{2, q_{1}} & \ldots & Q_{2, q_{d}} \\
\vdots & \ddots & \vdots & \vdots & \ddots & \vdots \\
-P_{d, p_{1}} & \ldots & -P_{d, p_{d}} & P_{d, q_{1}} & \ldots & P_{d, q_{d}}
\end{array}\right)\left(\begin{array}{c}
C_{, q_{1}} \\
C_{, q_{2}} \\
\vdots \\
C_{, p_{d}}
\end{array}\right)=\left(\begin{array}{c}
0 \\
0 \\
\vdots \\
0
\end{array}\right) .
$$

From the fact that $\Phi:(q, p) \mapsto\left(Q_{1}, \ldots, Q_{d}, P_{1}, \ldots, P_{d}\right)$ is a diffeomorphism we have $\operatorname{det} A \neq 0$ (because $A=D \Phi \cdot \mathbb{E}$ where $\mathbb{E}$ is the symplectic matrix) and so $C(q, p)=C$, a constant. It remains to show that $C=1$. This comes from the fact that outside a compact set of $\mathbb{R}^{2 d}$ we have $\left(Q_{1}, \ldots, Q_{d}, P_{1}, \ldots, P_{d}\right)=\left(q_{1}, \ldots, q_{d}, p_{1}, \ldots, p_{d}\right)$ and so $\left\{q_{i}, q_{j}\right\}=\left\{p_{i}, p_{j}\right\}=$ 0 and $\left\{q_{i}, p_{j}\right\}=\delta_{i j}$ outside this compact set. From the fact that the Poisson brackets are (at least) continuous, it follows that we must have $C=1$.

\section{References}

[1] F. Cardin and C. Viterbo, Commuting Hamiltonians and Hamilton-Jacobi multi-time equations, Duke Math. J. 144(2) (2008), 235-284.

[2] Y.M. Eliashberg, A theorem on the structure of wave fronts and its application in symplectic topology, Funktsional. Anal. Prilozhen. 21(3) (1987), 65-72.

[3] M. Gromov, Pseudoholomorphic curves in symplectic manifolds, Invent. Math. 82(2) (1985), 307-347.

[4] V. Humilière, Hamiltonian pseudo-representations, Comment. Math. Helv. 84(3) (2009), 571-585.

Università degli Studi di Padova

Dipartimento di Matematica Pura e Applicata

Via Trieste, 63

35121 PADOVA

ITALY

E-mail address: cardin@math.unipd.it

E-mail address: svazzole@gmail.com

Received 04/14/2011, accepted 11/08/2011 